Revisión de tema

\title{
Conducto subvesicular, nomenclatura anatómica para el conducto de Luschka y su importancia clínica
}

\author{
Subvesical Ducts, the Anatomical Nomenclature for the Bile Ducts \\ of Luschka and its Clinical Importance
}

\section{Canal subvesicular, nomenclatura anatômica para o ducto de Luschka e sua importância clínica}

Yelson Alejandro Picón-Jaimes, MD.*
Javier Esteban Orozco-Chinome, MD. **
Diana María Calderón-Sierra, MD. ***
Jessica Stephanie Molina-Franky, MD. ****

\section{Resumen}

Introducción: El conducto subvesicular fue descrito por Hubert Luschka como pequeños ductos biliares derivados de la pared de la vesícula biliar. Algunos autores lo describen como un ducto que lleva el drenaje desde el parénquima hepático hasta la vesícula, otros utilizan el término describiéndolo como un conducto que drena desde el parénquima hepático hasta las vías biliares extrahepáticas. Se planteó una revisión bibliográfica de la descripción anatómica del epónimo conducto de Luschka y de la terminología anatómica propuesta conducto subvesicular o subvesical, dada su importancia clínica como causa de fuga biliar. Metodología: Se realizó una revisión bibliográfica en bases de datos y bibliotecas electrónicas. Con ventana de tiempo personalizada desde 2006 hasta 2016, se obtuvo un total de 82 artículos, posteriormente se realizó un resumen analítico especializado, seleccionando un total de 46. Resultados: No se encontró consenso en la descripción anatómica del epónimo, ni en la descripción de la terminología internacional. Las lesiones de la vía biliar durante la colecistectomía se presentan como una complicación poco frecuente $(0.1-1.5 \%)$; sin embargo, tienen repercusiones en varios ámbitos y una morbi-mortalidad considerable. Conclusiones: Se evidenció discrepancia en la nomenclatura utilizada para la descripción de estos conductos, puesto que se denomina de manera indiscriminada como conducto de Luschka a todos los ductos biliares que se encuentran en la fosa biliar. La relevancia clínica radica en que es la segunda causa de fuga biliar iatrogénica y la asociación entre el conducto subvesical y carcinomas ductales. Aún se necesitan más estudios principalmente locales para identificar su incidencia y prevenir complicaciones. [Picón-Jaimes YA, OrozcoChinome JE, Calderón-Sierra DM, Molina-Franky JS. Conducto subvesicular, nomenclatura anatómica para el conducto de Luschka y su importancia clínica. Revisión de tema. MedUNAB 2017; 20(1): 54-62].

Palabras Clave: Conductos Biliares; Sistema Biliar; Conductos Biliares Extrahepáticos; Enfermedades de los Conductos Biliares; Procedimientos Quirúrgicos del Sistema Biliar; Neoplasias de los Conductos Biliares.

\footnotetext{
* Médico, Clínica Chía, Cundinamarca, Chía, Colombia.

** Médico, Hospital Universitario San Ignacio, Cundinamarca, Bogotá, Colombia

*** Médico, Residente de Cirugía General, Hospital Universitario San José, Cundinamarca, Bogotá, Colombia.

****Médico, Docente de la Universidad de Boyacá, Boyacá, Tunja, Colombia.
}

Correspondencia: Yelson Alejandro Picón Jaimes. Carrera 18 No. 113-52, Edificio Puerto Madero, San Patricio, Bogotá, Colombia. Teléfono: +57 3105747525. Email: ypicon@unab.edu.co 


\section{Abstract}

Introduction: The duct was described by Hubert Luschka as small bile ducts derived from the wall of the gallbladder. Some authors describe it as a duct leading to drainage from the hepatic parenchyma to the gallbladder; others use the term to describe it as a conduit draining from the hepatic parenchyma to the extrahepatic bile ducts. This article presents a literature review of the anatomical description of the eponymous Luschka conduit and the proposed anatomical terminology subvesicular or subvesical conduit, given its clinical importance as a cause of biliary leakage. Methodology: A bibliographic review was carried out in databases and electronic libraries. With a customized time window from 2006 to 2016, obtaining a total of 62 articles, a specialized analytical summary was subsequently performed, selecting a total of 46. Results: No consensus was found in the anatomical description of the eponym, nor in the description of the International terminology. Lesions of the biliary tract during cholecystectomy present as a rare complication (0.1 $1.5 \%$ ) but have repercussions in several areas and considerable morbidity and mortality. Conclusions: There was a discrepancy in the nomenclature used for the description of these ducts. It is indiscriminately named as the luschka's conduit for all bile ducts found in the biliary fossa. The clinical relevance is that it is the second cause of iatrogenic biliary leakage and the association between the subvesical duct and ductal carcinomas. More local studies are still needed to identify its incidence and prevent complications. [Picón-Jaimes YA, Orozco-Chinome JE, Calderón-Sierra DM, Molina-Franky JS. Subvesical Ducts, the Anatomical Nomenclature for the Bile Ducts of Luschka and its Clinical Importance. Topic Review. MedUNAB 2017; 20(1): 54-62].

Keywords: Bile Ducts; Biliary Tract; Bile Ducts, Extrahepatic; Bile Duct Diseases; Biliary Tract Surgical Procedures; Bile Duct Neoplasms.

\section{Introducción}

El anatomista Hubert von Luschka nació en Konstanz al sur de Alemania, su libro de 3 volúmenes de anatomía clínica se publicó entre 1862 y 1867 , y fue el primero en describir el estudio anatómico realizado en cadáveres humanos aparentemente sanos, mediante cortes y técnicas de conservación en los especímenes. Dentro de la descripción de estructuras anatómicas que cuentan con la denominación de su epónimo se describen el foramen de Luschka en el encéfalo, el nervio esfeno-nasal de Luschka y las criptas y los ductos de Luschka en la vesícula biliar(1).

Durante años, estas estructuras anatómicas fueron nombradas por su epónimo hasta que surgió el rechazo a esta nomenclatura dado que su denominación no "sigue la lógica propia de los signos lingüísticos, ni posee la sistematicidad y transparencia propia de los formantes grecolatinos que constituyen la fuente primaria de la cual se alimenta la terminología médica"' $(2,3)$.

\section{Resumo}

Introdução: $O$ ducto subvesicular foi descrito por Hubert Luschka como pequenos ductos biliares derivados da parede da vesícula biliar. Alguns autores descrevem isso como um ducto que leva a drenagem do parênquima hepático para a vesícula biliar, outros usam o termo para descrevê-lo como um canal que drena do parênquima hepático para os ductos biliars extra-hepáticos. Foi proposta uma revisão bibliográfica da descrição anatômica do canal epônimo de Luschka e da derivação anatômica proposta subvesicular ou subvesical, dada sua importância clínica como causa de vazamento biliar. Metodologia: foi realizada uma revisão bibliográfica em bancos de dados e bibliotecas eletrônicas. Com uma janela de tempo personalizada de 2006 a 2016, foi obtido um total de 82 artigos, seguido de um resumo analítico especializado, selecionando um total de 46. Resultados: Nenhum consenso foi encontrado na descrição anatômica do epônimo, nem na descrição da terminologia internacional. As lesões do trato biliar durante a colecistectomia apresentam-se como uma complicação rara (0.1 - 1.5\%); no entanto, têm repercussões em várias áreas e considerável morbidade e mortalidade. Conclusões: houve uma discrepância na nomenclatura utilizada para a descrição desses ductos, uma vez que é indiscriminadamente referido como o canal de Luschka a todos os canais biliares encontrados na fossa biliar. A relevância clínica é que é a segunda causa de vazamento biliar hepático e a associação entre ducto subvesical e carcinomas ductais. Mais estudos locais ainda são necessários para identificar sua incidência e prevenir complicações. [Picón-Jaimes YA, Orozco-Chinome JE, Calderón-Sierra DM, Molina-Franky JS. Canal subvesicular, nomenclatura anatômica para o ducto de Luschka e sua importância clínica. Revisão do tema. MedUNAB 2017; 20(1): 54-62].

Palavras-chave: Ductos Biliares; Sistema Biliar; Ductos Biliares Extra-Hepáticos; Doenças dos Ductos Biliares; Procedimentos Cirúrgicos do Sistema Biliar; Neoplasias dos Ductos Biliares.

El término conducto de Luschka fue descrito por el autor como estructuras tubulares en relación con la pared de la vesícula biliar, por su característica de ser epónimo ha adoptado diferentes descripciones anatómicas que obedecen a los errores más comunes escritos en la literatura como la polisemia, entendida como el significado múltiple de una palabra ya que, a través del tiempo, se presentan ampliaciones sucesivas de un concepto inicial, y la sinonimia entendida como el proceso mediante el cual un referente adquiere varios nombres no relacionados históricamente; en consecuencia, el término conducto de Luschka se ha designado para describir a cualquier conducto que se encuentre en la región biliar que no pertenezca directamente a una variación de la vía biliar extra hepática $(3,4)$.

La prevalencia mundial del conducto de Luschka corresponde a un tercio de la población mundial(5-7), otros autores la reportan del $0.5 \%$ al $50 \%(7-13)$ y la prevalencia del conducto subvesicular se estima corresponde del $4 \%$ al $10 \%$ (14). Se desconoce la prevalencia de dicha variante en nuestra población(4). 
La frecuencia de lesiones de vía biliar oscila entre un 0.1 a 0.6 $\%$, estas lesiones son entre tres y cuatro veces más frecuente durante la colecistectomía laparoscópica $(0.3-0.6 \%)$ que la abierta $(0.1-0.3 \%)(4)$.

La mayor parte de las lesiones de la vía biliar se deben a una mala interpretación de la anatomía, así como a la presencia de variantes anatómicas. La vesícula biliar puede tener posiciones anormales, ser intrahepática, rudimentaria, tener formas anómalas o estar duplicada (4). Este fenómeno y, en particular la descripción anatómica del epónimo conducto de Luschka y de la terminología anatómica propuesta conducto subvesicular o subvesical, será el objetivo principal de esta revisión, dada su importancia clínica como causa de fuga biliar, puesto que el procedimiento quirúrgico en las colecistectomías laparoscópicas y el tratamiento adecuado dependen de la precisión de la descripción anatómica.

\section{Metodología}

Se realizó una búsqueda sistemática (15) sobre el uso del epónimo conducto de Luschka para referirse a los conductos subvesicales o subvesiculares. La búsqueda de la literatura relacionada con el tema de estudio se planteó a partir de artículos de revisión, artículos originales, revisiones sistemáticas y revisiones de casos publicados en las bases de datos y bibliotecas electrónicas como: Pubmed, Central, Scielo, Medline, Ovid y ProQuest. Con base en los Descriptores en Ciencias de la Salud (DeCS) y Medical Subject Headings (MeSH) se seleccionaron las palabras clave para la búsqueda en idiomas español e inglés: Conductos Biliares, Sistema Biliar, Conductos Biliares Extrahepáticos, Bile Duct Diseases, Biliary Tract y Surgical Procedures, Operative. Se obtuvo un total de 82 artículos, para después realizar un resumen analítico especializado, seleccionando un total de 46 que conformaron la base para realizar este manuscrito. Como criterios de inclusión se tuvieron en cuenta todos los artículos disponibles en texto completo libre, publicados entre 2006 a 2016 y escritos en idioma español o inglés, y se excluyeron los artículos duplicados (Figura 1).

\section{Resultados}

Se analizaron 46 artículos de los cuales 28 correspondían a artículos originales, 6 a revisiones de tema, una revisión

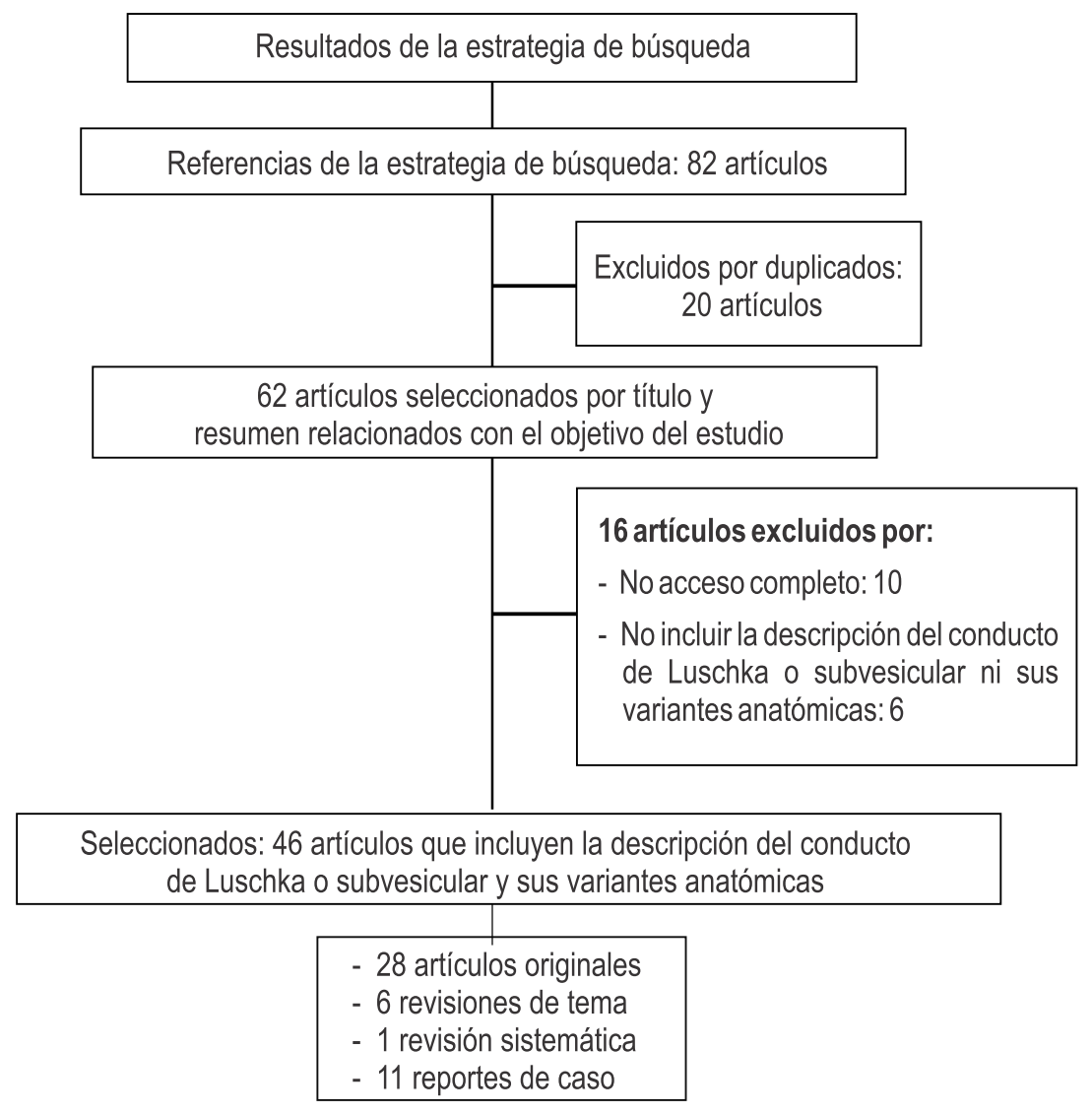

Fuente: Elaboración propia

Figura1. Resultados de la estrategia de búsqueda bibliográfica 
sistemática y 11 reporte de casos. En la tabla 1 se muestra el uso de la diferente terminología encontrada en cada manuscrito consultado (Tabla 1).

Del total de artículos revisados acerca de la nomenclatura anatómica internacional del conducto de Luschka se expondrán los resultados de acuerdo a la nomenclatura anatómica utilizada en cada fuente, partiendo del origen embriológico, las diferencias en nomenclatura y sus implicaciones clínicas obtenidas en la revisión.

\section{Embriología del conducto subvesical o subvesicular}

Se cree que los conductos de Luschka surgen de la proliferación anómala de los conductos biliares más distales formados a partir de la pars hepática a medida que se desarrolla en el tabique transverso (16). Estos conductos biliares pueden persistir en determinadas zonas cuando el parénquima hepático debe retroceder. En varios estudios practicados en fetos(17), se evidenció que la incidencia del conducto de Luschka es del 21\%, mayor que la encontrada en estudios realizados en adultos $(6,10,18)$.

\section{Conducto de Luschka versus nomenclatura internacional}

El médico alemán Hubert von Luschka, en el año de 1863, escribió un libro cuyo enfoque fue la descripción anatómica de órganos humanos como la vesícula biliar, el hígado y el tracto biliar, entre otros. Dentro de los apartados del libro mencionó dos estructuras tubulares en relación con la pared de la vesícula biliar y de los conductos biliares; la primera estructura dentro de sus características contiene glándulas intramurales que drenan en el lumen de la vesícula biliar a las cuales dio el nombre de criptas de Luschka; la segunda, es una estructura compuesta por pequeños conductos microscópicos localizados alrededor de la vesícula biliar poseedores de un epitelio cubital simple y dentro de ellos evidenció pigmento que sugería la presencia de bilis $(1,14)$.

Investigaciones recientes han descrito que son una región local de células progenitoras, que desempeñan un papel importante en el proceso de recuperación y renovación del epitelio del conducto biliar después de la lesión. Sin embargo, no se encuentra una definición por el anatomista que concuerde con las descripciones encontradas en la actualidad $(19,20,21)$.

\section{Descripción Anatómica del epónimo de Luschka en la actualidad}

En los artículos que utilizan el epónimo, se encontró unanimidad en la siguiente definición: El conducto de Luschka es un conducto biliar o varios conductos pequeños que miden aproximadamente de 1 a $2 \mathrm{~mm}$ de diámetro y se encuentran en la fosa vesicular, sin venas o arterias que lo acompañen en su recorrido $(6,10,22)$.

Diversos estudios han descrito variantes anatómicas como un conducto biliar que presenta el extremo proximal en el ducto hepático derecho y un extremo distal ciego(1); otros autores reportan un ducto biliar que drena el parénquima hepático derecho directamente a la vesícula biliar $(1,8,10,17,23-26)$; igualmente un conducto que inicia en el parénquima hepático derecho y termina en los conductos extra hepáticos derecho o común $(7,22)$ en otros cómo pequeños conductos biliares ubicados dentro de la fosa de la vesícula biliar (27), y finalmente se describe un conducto

Tabla1. Uso de terminología según los manuscritos consultados

\begin{tabular}{lr}
\hline Terminología & Número de artículos \\
\hline Uso de epónimo conducto de Luschka & 24 \\
\hline $\begin{array}{l}\text { Uso del término conducto subvesicular, } \\
\text { conducto aberrante hepático derecho y }\end{array}$ & 20 \\
conducto supravesical & 1 \\
\hline Conducto subvesical & 1 \\
\hline $\begin{array}{l}\text { Clasificación de Strasberg para lesiones de la } \\
\text { vía biliar como Tipo A la fuga del muñón cístico } \\
\text { o fuga de un conducto de Luschka }\end{array}$ & \\
\hline
\end{tabular}

Fuente: Elaboración propia 
que inicia en la vesícula biliar y se dirige al conducto hepático derecho $(8,9,12,13,28)$ (Figura 2).

\section{Definición anatómica de la nomenclatura internacional}

Como se escribió anteriormente, se ha encontrado un amplio uso del epónimo de Luschka acompañado por nomenclatura anatómica internacional, a continuación se citan las definiciones (tabla 2).

Conducto subvesical: Ducto biliar de 1 a $2 \mathrm{~mm}$ de diámetro que se encuentra localizado en la fosa vesicular y drena la bilis del lóbulo hepático derecho, pasando proximalmente a la vesícula biliar y drena en los conductos extra o intrahepáticos (5). Además, se encontró una definición adicional que los describe como conductos que inician en la pared de la vesícula y cruzan el parénquima del hígado por


Fuente: Elaboración propia

Figura 2. Descripción anatómica de los conductos subvesiculares conocidos por el epónimo conductos de Luschka. Tipos de conducto Subvesicular. El No.1 corresponde a un conducto pequeño que se origina en el conducto hepático derecho con un extremo ciego, el No.2 a un conducto que drena el parénquima hepático derecho directamente a la vesícula biliar, el No.3 presenta origen proximal en la región posterior de la vesícula biliar drenando a los conducto extra hepáticos, el No.4 drena la bilis del parénquima hepático derecho directamente a los conductos extra hepáticos, el No.5 son pequeños conductos que inician en la adventicia de la vesícula biliar hasta la adventicia del parénquima hepático.

Tabla 2. Definición anatómica de la nomenclatura internacional

\begin{tabular}{ll}
\hline Nomenclatura & Descripción anatómica \\
\hline Conducto subvesical & $\begin{array}{l}\text { Ducto biliar de } 1 \text { a } 2 \mathrm{~mm} \text { de diámetro localizado en la fosa } \\
\text { vesicular que drena la bilis del lóbulo hepático derecho, pasando } \\
\text { proximalmente a la vesícula biliar y drena en los conductos extra } \\
\text { ointrahepáticos }\end{array}$ \\
\hline Conducto subvesicular & Conjunto de ductos que se ubican en la fosa biliar \\
\hline Conducto hepaticolecístico & $\begin{array}{l}\text { Drena la bilis del lóbulo derecho del hígado directamente a la } \\
\text { vesícula biliar }\end{array}$ \\
\hline Conducto subvesical aberrante & $\begin{array}{l}\text { Múltiples conductillos que se dirigen desde el tejido conectivo } \\
\text { del lóbulo derecho del hígado hasta el tejido conectivo de la } \\
\text { vesícula biliar sin penetrarla directamente }\end{array}$ \\
\hline Conducto de Luschka ( 1863) & $\begin{array}{l}\text { Estructura compuesta por pequeños conductos microscópicos } \\
\text { localizados alrededor de la vesícula biliar poseedores de un } \\
\text { epitelio cubital simple y dentro de ellos evidenció pigmento que } \\
\text { sugería la presencia de bilis }\end{array}$ \\
\hline Conducto de Lluschka (actual) & $\begin{array}{l}\text { conducto biliar o varios conductos pequeños que miden } \\
\text { aproximadamente de } 1 \text { a } 2 \text { mm de diámetro y se encuentran en la } \\
\text { fosa vesicular, sin venas o arterias que lo acompañen en su } \\
\text { recorrido }\end{array}$ \\
\hline
\end{tabular}

Fuente: Elaboración propia basado en los aportes de los diferentes autores citados en este manuscrito. 
el lóbulo derecho hasta alcanzar el conducto hepático derecho o común $(6,11,29)$.

Conducto subvesicular: Conjunto de ductos que se ubican en la fosa biliar, estos pueden ser superficiales y drenan en el segmento posterior del hígado al conducto biliar común(14).

Conducto hepaticolecístico: Drena la bilis del lóbulo derecho del hígado directamente a la vesícula biliar(14).

Conducto subvesical aberrante: Está compuesto por múltiples conductillos que se dirigen desde el tejido conectivo del lóbulo derecho del hígado hasta el tejido conectivo de la vesícula biliar sin penetrarla directamente(14).

Adicional a esto, en otros estudios (30,31) describieron el conducto hepático derecho aberrante; sin embargo, las imágenes corresponden al conducto de Luschka y reportaron una incidencia del $8 \%$ de fuga biliar en colecistectomía (Figura 2).

\section{Importancia clínica}

Las lesiones de la vía biliar durante la colecistectomía se presentan como una complicación poco frecuente $(0.1 \%$ $1.5 \%)$. Estas lesiones son entre tres y cuatro veces más comunes durante la colecistectomía laparoscópica (0.3$0.6 \%$ ) que la abierta (0.1-0.3\%). Sin embargo, estas tienen repercusiones clínicas, económicas, judiciales, laborales y sociales muy importantes, una morbi-mortalidad considerable, con aumento de los costos, la hospitalización y efectos a largo plazo sobre calidad de vida $(27,32,33)$. En Colombia se desconoce la frecuencia de este problema, ya que no existen estudios de consenso.

Dentro de las causas se pueden evaluar aquellas condicionadas a la patología local, factores técnicos puros y por lo tanto dependientes del cirujano y las que nos competen en esta revisión: aquellas vinculadas a la disposición anatómica. Diferentes series de casos, demuestran que las lesiones durante la colecistectomía laparoscópica se reconocen usualmente en el procedimiento o más comúnmente en el postoperatorio inmediato $(27,34)$. En el postoperatorio inmediato la presencia de una bilirragia ya sea a través de un drenaje o signos peritoneales debe suponer una lesión biliar. La acumulación localizada de bilis puede producir un bilioma estéril, un absceso subhepático o interhepatofrénico. La ictericia en el postoperatorio inmediato tardío debe hacer sospechar siempre la presencia de lesión de via biliar primaria. La colangitis recidivante es otra forma de presentación de complicaciones. Solo en casos muy retrasados, el paciente tiende a manifestar cirrosis avanzada e hipertensión portal(4).

Especificamene las fugas biliares por conductos de Luschka usualmente no se diagnostican intraoperatoriamete, puesto que el efecto de la anestesia disminuye el drenaje de bilis; por lo tanto, suele sospecharse en la primera semana pos operatoria. La fuga biliar depende de la conexión y aumenta si hay obstrucción en los conductos hepáticos (22).

En la literatura clínica, se reporta una incidencia de fuga biliar pos colecistectomía laparoscopia de $0.4 \%$ a $25 \%$ $(14,24,35)$; considerando así, que la fuga biliar por los conductos subvesiculares son la segunda causa de fuga biliar, provocando formación de biliomas y bilioperitoneo que llegan a producir la muerte del paciente si no se tratan de manera adecuada $(6,7,9,11,12,14,24,25,32,36-43)$.

Otra patología asociada son los adenocarcinomas invasivos, puesto que, se han descrito seis casos de proliferación de los conductos de Luschka que imitan un adenocarcinoma invasivo de vesícula biliar, explicado porque los conductillos demostraron un patrón de crecimiento irregular, pérdida de la característica concéntrica, fibrosis y atipia epitelial que sugería fuertemente el diagnóstico de adenocarcinoma o adenocarcinoma metastásico que involucra la capa serosa de la vesícula biliar. Todos los casos se asociaron con episodios agudos y crónicos de colecistitis con ulceración de la mucosa, colelitiasis y engrosamiento de la pared vesicular. Este diagnóstico debe ser concluido después de una revisión exhaustiva del caso clinico, puesto que sí se han reportado casos de trasformación neoplásica en los ductos de Luschka como adenocarcinomas ductales $(6,8,9,44)$.

\section{Tratamiento adecuado dependiente de la descripción anatómica}

A las fístulas biliares provenientes del conducto cístico, el conducto biliar común y los conductos subvesiculares o de Luschka que se detectan en el posoperatorio y confirmado por tomografía computarizada o ecografía abdominal, se les recomienda el drenaje guiado por imágenes o el uso de un catéter como drenaje externo. Los casos de baja filtración biliar responden bien con la descompresión del conducto biliar mediante la colocación endoscópica de una prótesis o una sonda nasobiliar teniendo una tasa de éxito del $80-100 \%$ (38).

En el caso de detectar las fugas intra-operatoriamente mediante la colangiografia intra operatoria, se recomienda la ligadura o clipaje dependiendo del tamaño del conducto; sin embargo, hay que considerar la descompresión mediante el retiro de los cálculos en la vía biliar extra hepática, puesto que, si el origen de la fuga es del conducto subvesical que se comunica con el árbol biliar central y este se encuentra afectado por una obstrucción el drenaje de bilis será mayor. En estos casos, la colangiopancreatografía retrógrada endoscópica (CPRE) con esfinterotomía y la colocación de stents son el mejor método de diagnóstico y tratamiento dado que es un método relativamente simple y eficaz para este tipo de lesión, con tasas de éxito de $70 \%$ - $95 \%$ $(20,22,32,34,38)$. 
Algunos autores que recomiendan la realización de CPRE con esfinterectomía, mencionan la importancia de la descripción anatómica de la lesión, debido a que un conducto final ciego con conexión al conducto hepático derecho o común puede ser descomprimido mediante la CPRE, mientras que una lesión en un conducto biliar que drena a un lecho aislado que hace parte del parénquima hepático no puede ser tratado de esta manera $(1,7,8,13,21,24,43,45,46)$.

Tanto la naturaleza como las consecuencias de las lesiones durante la colecistectomía varían ampliamente, así como su pronóstico. La mortalidad global como consecuencia de lesión de vía biliar se presenta en un $2-5 \%(21,33)$. La mortalidad operatoria se encuentra entre el 5 al $8 \%$. La causa de muerte se debe a: hemorragia digestiva, insuficiencia hepatocítica e insuficiencia renal. Blumgart comunicó que en un total de 84 pacientes sometidos a reparación de la estenosis, la mortalidad a los 30 días fue del $2.4 \%$. No se especifica en los estudios analizados relación concreta entre las vaiantes anatómicas y el prónostico(4).

\section{Conclusiones}

El inadecuado uso de la terminología anatómica para referirse a los conductos subvesiculares genera dificultad tanto en el aprendizaje de la morfología para los estudiantes de ciencias básicas como para los médicos, razón por la cual puede incurrirse en errores graves al denominar los ductos que sin ser accesorios aparecen en la vía biliar.

Es imperativo tener un concepto muy claro sobre la presentación clínica, diagnóstico, clasificación, complejidad y tratamiento adecuado; pero sobre todo, la manera de prevenir las complicaciones en las diferentes variantes anatómicas para lo cual es relevante el adecuado uso de una nomenclatura universal, y el conocimiento estricto de las diferentes variantes anatómicas. Por lo tanto, el término conductos subvesiculares permite una mejor ubicación espacial al denominar a los pequeños ductos biliares localizados en la fosa biliar. Sin embargo, es tangible la ausencia de estudios en el ámbito local, seguimientos claros y escalas para seguimiento pronóstico de las complicaciones.

\section{Conflicto de intereses}

Los autores declaran no tener conflictos de interés.

\section{Referencias}

1. Boonstra EA, Lorenz K, Porte RJ. The Quest for Luschka's Duct: An Eponym Leading a Life of Its Own. Dig Surg [Internet]. 2014;31(2):104-7. Available from: http://www.karger.com?doi=10.1159/000362095
2. Goic G A. Sobre el uso de epónimos en medicina. Rev Med Chil [Internet]. 2009 Nov [cited 2017 Jan 19];137(11):1508-10. Available from: http://www. scielo.cl/scielo.php?script=sci arttext\&pid=s003498872009001100016\&lng=es\&nrm=iso\&tlng=es

3. Alcaraz Ariza M de los Á. Los epónimos en medicina. Ibérica [Internet]. 2002;4:55-73. Available from: http://www.aelfe.org/documents/text4-Alcaraz.pdf

4. Valsangiacomo P. Lesiones quirurgicas de via biliar. Facultad de Medicina, Hospital Maciel. Universidad de la República; 2014.

5. Majumder S, Habibi H, Garcia CM. Subvesical Bile Duct Injury. Surg Laparosc Endosc Percutan Tech [Internet]. 2013 Aug;23(4):e168-9. Available from: http:// dx.doi.org/10.1097/SLE.0b013e31828b8364

6. Kocabiyik N, Yalcin B, Kilbas Z, Karadeniz SR, Kurt B, Comert A, et al. Anatomical assessment of bile ducts of Luschka in human fetuses. Surg Radiol Anat [Internet]. 2009 Aug 12;31(7):517-21. Available from: http://link.springer.com/10.1007/s00276-009-0473-3

7. Spanos CP, Syrakos T. Bile leaks from the duct of Luschka (subvesical duct): a review. Langenbeck's Arch Surg [Internet]. 2006 Sep 23;391(5):441-7. Available from: http://link.springer.com/10.1007/s00423-006-0078-9

8. Neumann H, Fry L, Malfertheiner P, Mönkemüller K. Bile Leak from the Duct of Luschka. Z Gastroenterol [Internet]. 2010 Feb 2;48(2):256-7. Available from: http://www.thieme-connect.de/DOI/DOI?10.1055/s0028-1109474

9. Jahan $M$, Xiao $P$, Go A, Cheema M, Hameed $A$. Intraductal and invasive adenocarcinoma of duct of Luschka, mimicking chronic cholecystitis and cholelithiasis. World J Surg Oncol [Internet]. 2009;7(1):4. Available from: http://wjso.biomedcentral.com/ articles/10.1186/1477-7819-7-4

10. Singhi AD, Adsay NV, Swierczynski SL, Torbenson M, Anders RA, Hruban RH, et al. Hyperplastic Luschka Ducts. Am J Surg Pathol [Internet]. 2011 Jun;35(6):88390. Available from: http://www.pubmedcentral.nih.gov/ articlerender.fcgi?artid=3568524\&tool=pmcentrez\&rende rtype=abstract

11. Maker VK, Guzman-Arrieta ED. Cognitive Pearls in General Surgery [Internet]. Cognitive Pearls in General Surgery. New York, NY: Springer New York; 2015. 1-473 p. Available from: http://link.springer.com/10.1007/9781-4939-1850-8

12. Ko K, Kamiya J, Nagino M, Oda K, Yuasa N, Arai T, et al. A Study of the Subvesical Bile Duct (Duct of Luschka) in Resected Liver Specimens. World J Surg [Internet]. 2006 Jul 23;30(7):1316-20. Available from: http://link.springer.com/10.1007/s00268-005-0469-z

13. Pioche M, Ponchon T. Management of bile duct leaks. J Visc Surg [Internet]. 2013 Jun;150(3):S33-8. Available from: http://www.ncbi.nlm.nih.gov/pubmed/23791984

14. SchnelldorferT, Sarr MG, Adams DB. What is the Duct of Luschka?-A Systematic Review. J Gastrointest Surg [Internet]. 2012 Mar 4;16(3):656-62. Available from: http://link.springer.com/10.1007/s11605-011-1802-5

15. Merino-trujillo A. Como escribir documentos científicos (Parte 3). Artículo de revisión. Salud en Tabasco. 2011;17(1 y 2):36-40.

16. Sutton ME, Op den Dries S, Koster MH, Lisman T, Gouw $\mathrm{ASH}$, Porte RJ. Regeneration of human extrahepatic 
biliary epithelium: The peribiliary glands as progenitor cell compartment. Liver Int. 2012;32(4):554-9.

17. Mitidieri V, Ottone NE. La Vía Biliar Intrahepática: Clasificación Anátomo-Quirúrgica con Base Colangiográfica. Int J Morphol [Internet]. 2015 Dec;33(4):1427-35. Available from: http://www.scielo.cl/scielo.php?script=sci_arttext\&pid=S 0717-95022015000400039\&lng=en\&nrm=iso\&tlng=en

18. Doumenc $B$, Boutros $M$, Dégremont $R$, Bouras $A F$. Biliary leakage from gallbladder bed after cholecystectomy: Luschka duct or hepaticocholecystic duct? Morphologie [Internet]. 2016;100(328):36-40. A $\vee$ a i I a b I e f r o m : http://dx.doi.org/10.1016/j.morpho.2015.08.003

19. Stewart L, Way LW. Laparoscopic bile duct injuries: Timing of surgical repair does not influence success rate. A multivariate analysis of factors influencing surgical outcomes. Hpb. 2009;11(6):516-22.

20. Hii MW, Soylu A, Aygun E. The role of ERCP in the management of bile leakage: endoscopic sphincterotomy versus biliary stenting. J Laparoendosc Adv Surg Tech A. 2010;20(5):455-9.

21. Parlak E, Disibeyaz S, Odemis B, Koksal AS, Kucukay F, Sasmaz N, et al. Endoscopic treatment of patients with bile duct stricture after cholecystectomy: factors predicting recurrence in the long term. Dig Dis Sci. 2015;60(6):1778-86.

22. Boleko Ribas M de los ángeles. Estudio retrospectivo de las lesiones iatrogénicas de la vía biliar durante la era laparoscópica en un hospital de tercer. Universidad Autónoma de Barcelona; 2011.

23. Lau WY, Lai ECH, Lau SHY. Management of bile duct injury after laparoscopic cholecystectomy: a review. ANZ J Surg [Internet]. 2010 Jan;80(1-2):75-81. Available from: http://doi.wiley.com/10.1111/j.1445-2197.2009.05205.x

24. Soria M, del Valle R, Bravo G, Ospina J, RoblesMedranda C. ¿Es la esfinterectomía con prótesis plástica por 8 semanas suficiente para tratar las fugas biliares ? Endoscopia [Internet]. 2015 Jan;27(1):25-30. Available from: http://linkinghub.elsevier.com/ retrieve/pii/S0188989315000123

25. Parampalli U, Helme S, Asal G, Sinha P. Accessory cystic duct identification in laparoscopic cholecystectomy. Gd Rounds. 2008;8:40-2.

26. Díaz-Chiclayo AL, Rodríguez-Gastelo J. Anestesia general comparada con la anestesia epidural en la colecistectomía laparoscópica. Rev cuerpo méd [Internet]. 2013;6(1):37-40. Available from: http://sisbib.unmsm.edu.pe/BVRevistas/cuerpomedico hnaaa/v6n1 2013/pdf/a08v6n1.pdf

27. Rajab R, Meara N, Chang F. Florid Ducts of Luschka Mimicking a Well Differentiated Adenocarcinoma of the Gallbladder : A Case Report. The Int J Pathology. 2007;6(1):1-5.

28. Russo AM, Sciuto PM. Conducto biliar subvesicular: Hallazgo quirúrgico y colangiográfico. Rev Arg de Anat Clin. 2016;8(1):50-4.

29. Watanabe M, Shiozawa K, Kishimoto Y, Mimura T, Ito K, Kamata I, et al. Duct of Luschka diagnosed by sonography in a patient with bile duct carcinoma and intrahepatic bile duct dilatation. J Clin Ultrasound [Internet]. 2013;41(9):558-62. Available from: http://doi.wiley.com/10.1002/jcu.21998
30. González RR, Alfonso MÁM, Peña RT, Milhet ABL, Fraga JGP, González JB. Reintervenciones por complicaciones después de realizada colecistectomía laparoscópica. Rev Cuba Cir. 2011;50(3):302-11.

31. Elmunzer BJ, Taylor JR. Aberrant right hepatic duct with patent ducts of Luschka. Gastrointest Endosc [Internet]. 2011;74(1):196-7. Available from: http://dx.doi.org/ 10.1016/j.gie.2011.02.025

32. Booij K, de Reuver P, Yap K, van Dieren S, van Delden O, Rauws E, et al. Morbidity and mortality after minor bile duct injury following laparoscopic cholecystectomy. Endoscopy [Internet]. 2014 Dec 22;47(1):40-6. Available from: http://www.thieme-connect.de/DOI/ DOI?10.1055/s-0034-1390908

33. Ibañez L, Escalona A, Devaud J N, Montero M P, Ramírez W E, Pimentel F. Colecistectomía laparoscópica: experiencia de 10 años en la Pontificia Universidad Católica de Chile * Laparoscopic cholecystectomy : 10 years at the Hospital Clínico. Rev Chil Cir. 2007;59(May 2001):10-5.

34. Hii MW, Gyorki DE, Sakata K, Cade RJ, Banting SW. Endoscopic management of post-cholecystectomy biliary fistula. Hpb. 2011;13(10):699-705.

35. Kim KH, Kim TN. Endoscopic Management of Bile Leakage after Cholecystectomy: A Single-Center Experience for 12 Years. Clin Endosc [Internet]. 2014;47(3):248. Available from: http://ece.org/journal/view.php?doi=10.5946/ce.2014.47.3.248

36. Fasoulas K, Zavos C, Chatzimavroudis G, Trakateli C, Vasiliadis T, loannidis A, et al. Eleven-year experience on the endoscopic treatment of post-cholecystectomy bile leaks. Ann Gastroenterol. 2011;200-5. Available from: www.annalsgastro.gr

37. Machado NO. Biliary Complications Post Laparoscopic Cholecystectomy: Mechanism, Preventive Measures, and Approach to Management: A Review. Diagn Ther Endosc [Internet]. 2011;2011:1-9. Available from: http://www.hindawi.com/journals/dte/2011/967017/

38. Hernández AG, Gabriel C, Vega B, Díaz M, Ponce DL, Carlos J, et al. Resultados del tratamiento endoscópico de fístulas biliares posoperaytorias en el en el Hospital General de México. Endoscopia. 2011;XXV(4):211-7.

39. Tantia O, Jain M, Khanna S, Sen B. latrogenic biliary injury: 13,305 cholecystectomies experienced by a single surgical team over more than 13 years. Surg Endosc [Internet]. 2008 Apr 18;22(4):1077-86. Available from: http://link.springer.com/10.1007/s00464-0079740-8

40. Pinkas H, Brady PG. Biliary leaks after laparoscopic cholecystectomy: Time to stent or time to drain. Hepatobiliary Pancreat Dis Int. 2008;7(6):628-32.

41. Stewart L, Way LW. Laparoscopic bile duct injuries: Timing of surgical repair does not influence success rate. A multivariate analysis of factors influencing surgical outcomes. Hpb. 2009;11(6):516-22.

42. Hoyos C, Original Q, Colomb VMR. Lesión quirúrgica de la vía biliar : experiencia en la Unidad de Cirugía Hepatobiliar y Pancreática del Hospital Pablo Tobón Uribe de Medellín. Rev Colomb Cir. 2009;24:244-9.

43. Zhi-bing O, Sheng-wei L, Chang-an L, Bing T, Chuan-xin W, Ding $X$, et al. Prevention of common bile duct injury during laparoscopic cholecystectomy. Hepatobiliary Pancreat Dis Int. 2009;8(4):414-7. 


\section{Med}

UNAB Yelson Alejandro Picón-Jaimes, Javier Esteban Orozco-Chinome, Diana María Calderón-Sierra, Jessica Stephanie Molina-Franky

Vol. 20(1):54-62, abril - julio 2017

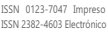

44. Dixon E. Management of Benign Biliary Stenosis and Injury [Internet]. Dixon E, Vollmer CM, May GR, editors. Cham: Springer International Publishing; 2015. Available from: http://link.springer.com/10.1007/978-3319-22273-8

45. Mori S, Kasahara M. Papillary adenocarcinoma of the subvesical duct. J Hepatobiliary Pancreat Surg. $2001 ; 8(5): 494-8$.

46. Fischer CP, Fahy BN, Aloja TA, Bass BL, Gaber AO, Ghobrial RM. Timing of referral impacts surgical outcomes in patients undergoing repair of bile duct injuries. Hpb. 2009;11(1):32-7. 